\title{
Measurement of striated muscle fibre diameters using interactive computer-aided microscopy
}

\author{
G SLAVIN, C SOWTER, PWARD, K PATON \\ From Northwick Park Hospital and Clinical Research Centre, Harrow'
}

SUMMARY We have measured muscle fibre diameters using two methods of interactive computer-aided microscopy. They are simple to perform, reproducible and more convenient than manual methods of measurement. The technique is of general application to histological measurement.

Measurement of muscle fibre size is important in the assessment of neuromuscular disease, and when combined with enzyme histochemistry demonstrates selective injury of the different muscle fibre types to various insults.' However, widespread use of muscle fibre measurements is restricted by the difficulties of measurement which are reflected in the numerous methods described to record different parameters and by the effort needed in many methods to make the measurement. The cross-sectional area of individual fibres has been assessed. ${ }^{2.3}$ Others have preferred to measure fibre diameters. Most fibres in cross section are circular or ellipsoid. It has been suggested that orthogonal diameters (major and minor axes) should be measured and the biaxial diameter ratio used as the appropriate measurement. ${ }^{4}$ However, many workers ${ }^{5-7}$ measure only a single diameter in one plane assuming that each fibre is cylindrical and of circular cross section.

To estimate the size of the fibre it is necessary to estimate the diameter of a circular cylinder cut by the arbitrary plane of the section. If a circular cylinder of diameter $D$ is cut by any plane then the plane section is an cllipse of minor axis D. Hence fibre diameter can be measured by treating each fibre section in whatever plane it is cut as an ellipse and measuring its minor axis. In practice the greatest diameter across the longest chord is measured. ${ }^{6}$

Practical techniques of measurement have included direct measurement using an eye piece micrometer measurement of a projected image or enlarged microphotographs. Recently computer-aided microscopy has been applied to this problem and methods have been described using either automatic image analysis to measure muscle cross sectional areas ${ }^{2.3}$ or interactive techniques. ${ }^{8}$ However. methods based on cross sectional area are unsatisfactory for many small biopsies, because obliquely cut fibres must be ignored. Moreover. automa-

Accepted for publication 12 May 1982 tic image analysis of fibres stained selectively by the ATPase reaction requires time-consuming editing, particularly to allow the adequate differentiation of light type 1 fibres from the background connective tissue.?

We have approached the problem of measurement of muscle fibres by choosing to measure the diameter of the fibres as described by Brooke and Engel ${ }^{5}$ but using computer-assisted microscopy with pathologist interaction to define the fibre type. We have compared the values obtained by computer-aided microscopy with those obtained by direct measurement in enlargeg photographic prints which was the method previous used in our laboratory.

\section{Material and methods}

The material examined was striated muscle from the vastus lateralis of 10 normal volunteer controls. 10 응 patients with hypothyroid myopathy and 10 patients with a variety of neuromuscular diseases. The tissue was orientated on cork to a transverse position using a dissecting microscope and snap-frozen with liquid ? nitrogen and isopentane. Sections were cut at $8 \mu \mathrm{m}$ and stained for ATPase at $\mathrm{pH} 9 \cdot 4$ to demonstrate the different fibre types. The diameter of type I and type II muscle fibres was then measured by three techniques: two methods based on computer-aided microscopy were compared with the manual method we used previously.

METHOD I (MANUAL METHOD)

The microscopic slide is photographed and the prints enlarged to a diameter of $\times 250$. The observer then covers the photograph with a sheet of translucent paper and records thereon with a pencil the longest chord perpendicular to the longest diameter of each fibre cross section. When all fibres have been so treated the record of this overlay shows a width vector for each fibre. The length of each vector is then measured and the appropriate width of the fibre estimated from the magnification. 


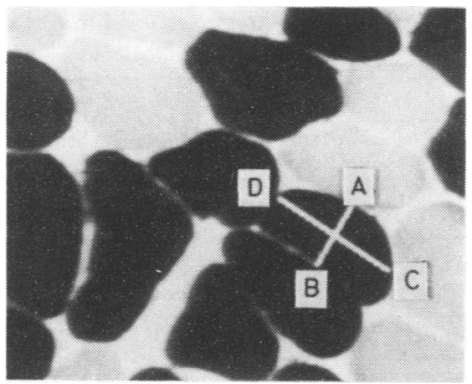

Fig. 1 The pathologist has selected line $A-B$ as the appropriate diameter. Line $C$-D lies along the longest axis and the selection is a good one.

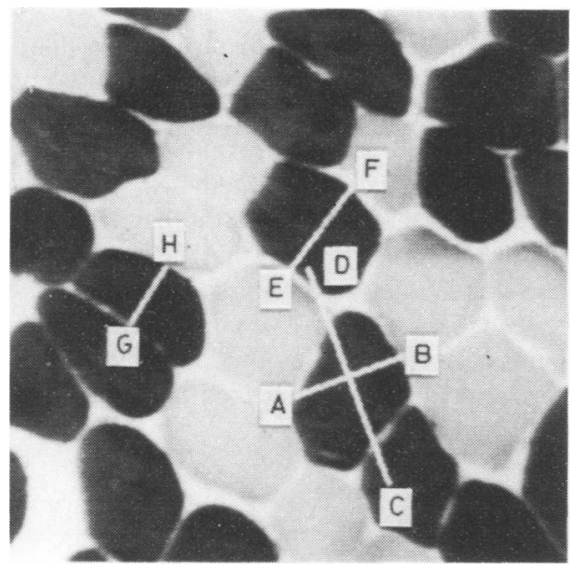

Fig. $2 E-F, G-H$ mark diameters measured and accepted. $A-B$ has been selected but line $C-D$ show's this to be erroneous and is now deleted.

METHODS $2 \& 3$ (COMPUTER-AIDED)

MICROSCOPY)

These methods used the Joyce Loebl "Magiscan" image analysis system. The Magiscan is based on a Data General Nova mini-computer and a microprocessor specialised for image analysis. A television camera records the microscopic image which is displayed on a monitor. A "light-pen" allows the observer to interact with image in accordance with a specific program written for each method.

\section{Method 2 ("Two point method")}

The observer views a televised image of the section on the monitor screen and using the light-pen indicates the two end points of the longest chord perpendicular to the longest diameter of each fibre section. If these end points are A. B, the computer display shows an unequal-armed cross made up of $A, B$ and $C$, D where the cross line $C D$ is the perpendicular bisector of $\mathrm{AB}$. If the observer had made a good choice then cross line $C D$ should lie along the longest diameter of the fibre (Fig. 1). If the choice is a bad one then the cross line is clearly seen to be at variance

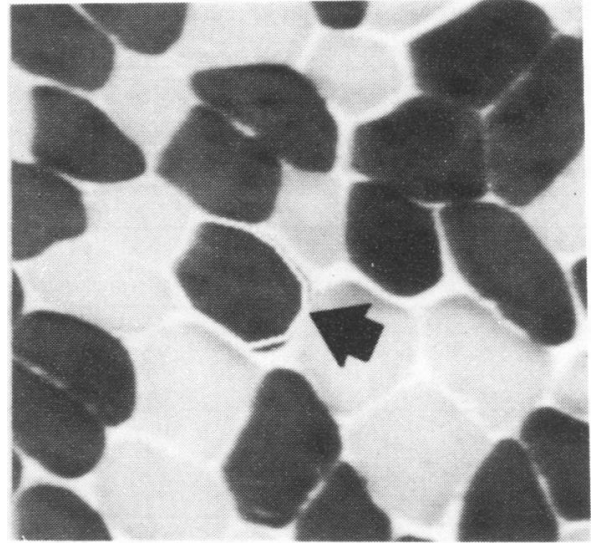

Fig. 3 The outline of the fibre is delineated with the light pen (arrow') and the axes of the "best ellipse" computed.

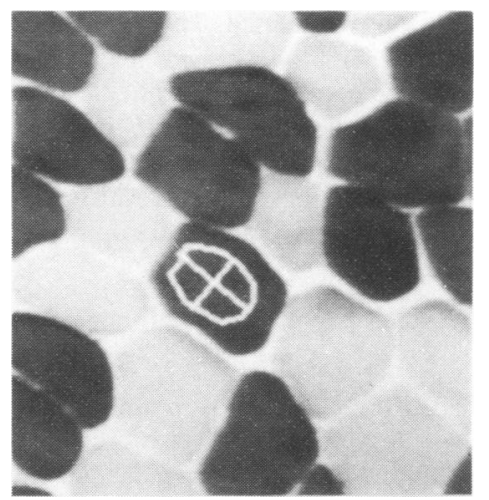

Fig. 4 A shrunken version of the outline and axes marks those fibres measured.

with the longest diameter (Fig.2). At this point, prompted by the vector display the observer accepts or rejects the line $A B$ and the appropriate width vector. If accepted the line $\mathrm{AB}$ remained as a marker on the display showing that the fibre has been measured. If the line $A B$ is rejected it is deleted and a new line selected. When the observer has dealt with all the fibres the pattern on the monitor screen is similar to that produced on the transparent overlay in the manual method.

\section{Method 3 ("Outline method")}

The observer views the microscopic image on the monitor of the Magiscan system and defines each fibre by drawing its outline with a light-pen on the televised image (Fig.3). The computer approximates to the given outline with the "best ellipse" which fits the outline and measures the minor axis of this ellipse as the muscle "diameter." After measurements a shrunken version of the major and minor 
Table 1 Differences in fibre size ( $\mu \mathrm{m})$ in fibre types, showing mean within-subject differences and standard deviation of individual differences between methods

\begin{tabular}{|c|c|c|c|c|c|c|}
\hline & \multicolumn{6}{|l|}{ Methods compared } \\
\hline & $\begin{array}{l}\text { Manual is outline } \\
\text { Mean }\end{array}$ & $S D$ & $\begin{array}{l}\text { Mamual istuopoint } \\
\text { Mean }\end{array}$ & $S D$ & $\begin{array}{l}\text { Outine is mopoint } \\
\text { Mean }\end{array}$ & $S D$ \\
\hline $\begin{array}{l}\text { Type I fibres } \\
\text { Type II fibres }\end{array}$ & $\begin{array}{l}1.9 \\
2.2 \\
\end{array}$ & $\begin{array}{l}3.2 \\
3.5\end{array}$ & $\begin{array}{l}1.6 \\
1.8\end{array}$ & $\begin{array}{l}3.6 \\
4.0\end{array}$ & $\begin{array}{l}-0.3 \\
-0.3\end{array}$ & $\begin{array}{l}2.7 \\
2.4\end{array}$ \\
\hline
\end{tabular}

Table 2 Mean within-subject differences and standard deviation of individual differences ( $\mu$ m) between methods

\begin{tabular}{|c|c|c|c|c|c|c|}
\hline & \multicolumn{6}{|l|}{ Merhods compared } \\
\hline & $\begin{array}{l}\text { Manual vs outline } \\
\text { Mean }\end{array}$ & $S D$ & $\begin{array}{l}\text { Manual istuopoint } \\
\text { Mean }\end{array}$ & $S D$ & $\begin{array}{l}\text { Oulline istwopoint } \\
\text { Mean }\end{array}$ & $S D$ \\
\hline Normal muscle & 0.7 & 3.2 & 1.8 & 4.2 & 1.1 & 3.3 \\
\hline Hypothyroid muscle & 3.9 & 3.2 & 3.1 & 3.9 & -0.8 & 1.8 \\
\hline Miscellaneous muscle disorders & 1.6 & 2.9 & 0.3 & 2.7 & -1.3 & 1.6 \\
\hline All groups & 2.0 & 3.3 & 1.7 & 3.8 & -0.3 & 2.5 \\
\hline
\end{tabular}

axes are displayed on each fibre as a record of completed measurement (Fig. 4).

In each method 150 fibres of both type I and type II from the biupsy were counted. The purpose of this study was to compare methods as used in practice. We have made no attempt to measure identical fibres and our results are based on sets of 150 fibres in fields chosen at random within the whole biopsy. In the computer-based methods the program presents the results as a histogram and calculates the atrophy and hypertrophy factors for the pathologist.

\section{Results}

We have made a direct comparison of the results obtained using each method. These methods were compared by (i) the mean within-subject difference, which measures overall agreement and (ii) the standard deviation of the individual differences, which measures between-subject variability around the mean value.

Table I shows that the mean and standard deviation of the differences between each pair of methods were very similar for type I and type II fibres and for the remaining analyses the two types were not distinguished, but were treated as different samples. Table 2 shows the comparison of the methods for the three groups of subjects; normals, those with hypothyroid myopathy and those with miscellaneous neuromuscular diseases, and for all groups combined.

Overall there was better agreement between the two computer-aided methods than between either of these and the manual method. Moreover, whereas for normals there was a similar degree of agreement between the manual $\stackrel{\overrightarrow{.}}{\overrightarrow{.}}$ method and the "two point" computer method with the $\overrightarrow{\vec{N}}$ "outline" computer method, for the pathological groups $\infty$ the hand method was rather worse than the "two point" method in its agreement with method 3. For all groups there were some samples where the hand method gave $z$ values that were very different from those obtained by the computer-aided methods. This is shown in Table 3 where it can be seen that for one in five samples the hand method gave a mean fibre diameter that was more than $5 \mu \mathrm{m}$ different from either computer method: usually the hand method gave a higher value.

\section{Discussion}

The value of measurement of changes in individual muscle fibres is well established in histological assess- $\mathbb{\mathbb { D }}$ ment of neuromuscular disease and allows the demonstra- $\vec{\overrightarrow{ }}$ tion of selective injury to metabolically vulnerable fibre $\frac{9}{3}$ types. The particular measurement made has varied but the most commonly used in clinical practice appears to be fibre type measurement as described by Brooke and Engel. ${ }^{5}$ The advent of computer-aided microscopy has? emphasised area measurements again because of the relative simplicity of simple grey level segmentation techniques. ${ }^{2.3}$ We have preferred to measure fibre diameters both to ensure comparability with other studies and also because obliquity of section distorts area 오 measurements and may make much of a small biopsy unsuitable. Moreover, ATPase staining for fibre differentiation is not always uniform either within a single $N$ fibre of between individual fibres of the same type.

Table 3 Extent of agreement (\%) between manual and computer-aided methods

\begin{tabular}{|c|c|c|c|}
\hline \multirow[b]{2}{*}{ Margin between methods $(\mu \mathrm{m})$} & \multicolumn{3}{|l|}{ Methods compared } \\
\hline & Manual is outline & Manual istwopoint & Outline vs twopoin \\
\hline $\begin{array}{l}2 \\
3 \\
4 \\
5 \\
\end{array}$ & $\begin{array}{l}33 \\
53 \\
70 \\
80\end{array}$ & $\begin{array}{l}42 \\
52 \\
70 \\
80 \\
\end{array}$ & $\begin{array}{l}55 \\
70 \\
87 \\
93 \\
\end{array}$ \\
\hline
\end{tabular}


"Automatic" methods still require time-consuming editing to account for vagaries of staining and particularly to delineate the light staining type I fibres if the analysis relies on grey level segmentation. Alternatively a major and expensive programming effort is necessary to produce a fully automatic routine.

The computer methods we described here are based on simple interaction between pathologist and computer. The pathologist uses his interpretative skill to delineate the picture for analysis; the computer then makes the appropriate measurements.

Both the computer-aided methods we describe are faster and more convenient than simple hand measurements on photographs. There is no "true" answer to such measurements and values obtained by hand measurement and by the "two point" method both depend on subjective decision by the pathologist of the longest fibre diameter. We have therefore compared these results with a more objective measure - the minor axis of the "best ellipse" fitting the outline which was obtained by tracing on the screen image of the fibre with the light-pen and requiring only minimal subjective decision by the pathologist.

Our results show that there is better agreement between the two computer methods than bet ween either computer method and manual measurement. However, the discrepancies between manual and computer methods are small and $80 \%$ of results are within $5 \mu \mathrm{m}$ of each other. We chose the "outline trace" as a more objective measure against which the other methods could be assessed. In working practice. however, it requires more operator effort and is less rapid than the "two point" method. Since the results obtained by the two computer methods do not differ significantly we prefer the "two point" method and it is in routine use in this laboratory. It is simple, rapid to perform and the prompting cross line ensures accuracy. This method has been rapidly learned by a variety of staff - students. medical laboratory scientific officers and clinicians - and is readily accepted by them. It is clearly applicable to the measurement of other tissue components and is a technique of general application.

We wish to thank Mr D Altman for his statistical advice and Mrs Jillian Pirie for her typescript.

Since writing this paper the software for the two-point method has been updated by Mr Roger Jagoe to facilitate counting of muscle subtypes within a single image.

\section{References}

'Engel WK Selective and non-selective susceptibility of muscle fibre types. Arch Neurol 1970:22:97-117.

2 Scott JWM. Joy J. the cross-sectional area of diaphragmatic muscle fibres in emphysema, measured by an automated image analysis system. J Pathol 1976:120:121-8.

3 Jaffe DM, Terry RD, Spiro AJ. Disuse atrophy of skeletal muscle. J Neurol Sci 1978:35:189-200.

${ }^{4}$ Song SK. Shimada N, Anderson PJ. Orthagonal diameters in the analysis of muscle fibre size and form. Nature 1963:200:1200-21.

${ }^{5}$ Brooke MH. Engel WK. The histographic analysis of human muscle biopsies with regard to fibre types. Neurology (Minneap) 1969: 19:221-34

${ }^{6}$ Dubowitz V. Brooke MH. Muscle biopsy: a modern approach. London WB Saunders. 1977.

${ }^{7}$ McKeran RO, Slavin G. Andrews TM. Ward P. Mair WPG. Muscle fibre type changes in hypothyroid myopathy. J Clin Pathol 1975; 28: 659-63.

${ }^{8}$ Jones DA, King MF, Round JM. Moppet. a rapid and accurate system for the measurement of muscle fibre cross-sectional areas In: Proceedings of Physiological Society, March 1980. J Physiol 1980; 305:5P.

Since this paper was submitted a further paper has appeared: Round JM. Jones DA. Edwards RHT. A flexible microprocessor system for the measurement of cell size. J Clin Pathol 1982:35:620-4.

Requests for reprints to: Dr G Slavin, Department of Histopathology, Northwick Park Hospital, Watford Road, Harrow. Middlesex HAl 3UJ, England. 